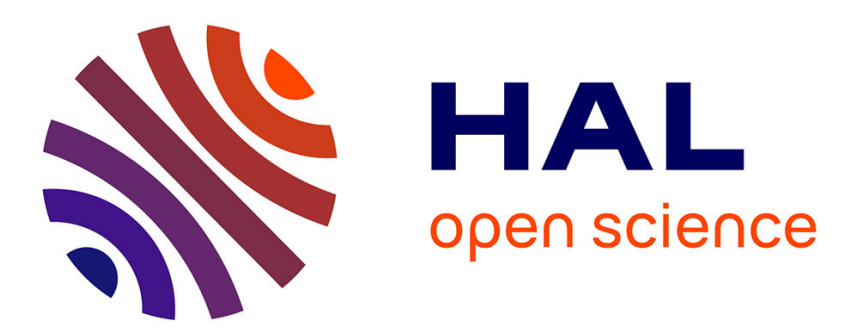

\title{
L'argent ne fait pas le bonheur. Réflexions autour du rapport de la commission Stiglitz
}

Emile Quinet

\section{To cite this version:}

Emile Quinet. L'argent ne fait pas le bonheur. Réflexions autour du rapport de la commission Stiglitz: Réflexions autour du rapport de la commission Stiglitz. 2010, pp.51-56. 10.1051/futur/36251 . halshs-00754456

\section{HAL Id: halshs-00754456 https://hal-pjse.archives-ouvertes.fr/halshs-00754456}

Submitted on 31 May 2020

HAL is a multi-disciplinary open access archive for the deposit and dissemination of scientific research documents, whether they are published or not. The documents may come from teaching and research institutions in France or abroad, or from public or private research centers.
L'archive ouverte pluridisciplinaire HAL, est destinée au dépôt et à la diffusion de documents scientifiques de niveau recherche, publiés ou non, émanant des établissements d'enseignement et de recherche français ou étrangers, des laboratoires publics ou privés. 


\title{
L'argent ne fait pas le bonheur
}

\author{
RÉFLEXIONS AUTOUR DU RAPPORT \\ DE LA COMMISSION STIGLITZ 1
}

PAR ÉMILE QuineT ${ }^{2}$

Émile Quinet nous livre ici une analyse du Rapport de la Commission sur la mesure des performances économiques et du progrès social, présidée par Joseph Stiglitz. Ainsi rappelle-t-il la pertinence des "critiques classiques au PIB (produit intérieur brut) ", dont il expose la teneur. Mais, tout en saluant, au-delà du rapport de synthèse, la " somme impressionnante des travaux scientifiques approfondis" engagés par la Commission, il s'étonne que celle-ci ait été composée exclusivement d'économistes et donc de la mise à l'écart des autres sciences sociales. $\mathrm{H} . J$.

T es récents travaux de la commisـ sion Stiglitz méritent l'attention à bien des égards, et d'abord par la solennité qui les a entourés. C'est le président de la République française qui a créé cette Commission, a participé à la séance publique de restitution de ses conclusions, organisée sous la forme d'une journée d'études dans le grand amphithéâtre de La Sor- bonne plein à craquer, et a prononcé un discours fort engagé dans le sens des thèses de la Commission. La composition de la Commission est également remarquable : elle ne comportait pas moins de cinq prix Nobel d'économie et une quinzaine d'autres économistes parmi les plus réputés au plan mondial concernant les sujets abordés.

I. STIGLITZ Joseph E., SEN Amartya et FITOUSSI Jean-Paul. Rapport de la Commission sur la mesure des performances économiques et du progrès social. Paris : ministère de l'Économie, de l'Industrie et de l'Emploi, 2009, 324 p.

2. Professeur émérite de l'École nationale des ponts et chaussées, et membre de l'Académie des technologies. 
La Commission avait pour tâche, comme le rappelle la synthèse du rapport, de « déterminer les limites du produit intérieur brut (PIB) en tant qu'indicateurs des performances économiques et du progrès social » et de « proposer de nouveaux indicateurs des performances économiques et de progrès social ». Elle consacre le quart de son texte au premier point, l'analyse critique du PIB, et le titre de cette partie est symptomatique : «Les critiques classiques au PIB ». Car les défauts du PIB en tant que mesure de la valeur de la production sont bien connus des spécialistes. On peut, à la suite du rapport, les classer en trois grandes catégories.

\section{Les limites du PIB}

D'abord, cet indicateur est limité à la production marchande. Les productions non marchandes (sécurité, santé, services publics....) sont mesurées non à leur produit, mais à leur coût monétaire, et il peut y avoir des écarts considérables entre les deux. Ainsi, les productions domestiques, dont le coût monétaire est nul, sont comptées pour zéro.

Ensuite, le PIB ne dit rien sur la qualité des biens et services, ni plus généralement sur la qualité de la vie qu'ils nous permettent d'avoir. Les dégradations des biens communs telles que les atteintes à l'environnement ne sont donc pas prises en compte, tout comme par exemple le caractère pénible ou dégradant de certaines formes de travail, ou encore le niveau d'éducation de la population. Dans le même ordre d'idées, les modifications du capital sont ignorées. Or, un PIB élevé peut résulter d'une réduction du capital, c'està-dire d'une perte de substance.
Enfin, dernière grande catégorie de critiques, le PIB ne dit rien non plus sur la répartition des revenus entre catégories sociales. Une augmentation du revenu national moyen peut s'accompagner d'une forte augmentation pour certaines catégories et d'une réduction pour d'autres. C'est d'ailleurs ce qui s'est produit, ces dernières années, dans la plupart des pays occidentaux, où il y a eu augmentation des inégalités et aggravation de la situation des plus pauvres, au moins en termes de revenus.

Sur cette base, le rapport explore les pistes de progrès pour élaborer de nouveaux indicateurs. Ses conclusions sont résumées dans un certain nombre de recommandations, I2 au total. Une petite moitié est destinée à corriger les calculs actuels du PIB pour les recentrer, non pas sur la source, la production, mais sur la destination finale, la consommation et les ménages : mieux intégrer les activités non marchandes, la répartition des revenus, le patrimoine et ses variations.

Les autres traitent des composantes du progrès qui dépassent le champ d'un produit intérieur brut même amélioré. Car l'une des conclusions du rapport, c'est qu'il ne peut pas y avoir un seul indicateur pour évaluer le progrès économique et social qui est multidimensionnel.

Plusieurs pistes sont ainsi recensées. La première concerne les évaluations du progrès à travers des indicateurs objectifs, comme la santé (espérance de vie) ou l'éducation, ou à travers des indicateurs d'opinion. Une autre consiste à porter l'attention sur les potentialités (les capacités et fonctionnalités selon les termes techniques consacrés) offertes par l'organisation sociale aux individus. Elle 
renvoie à des caractéristiques, telles que la liberté, la sécurité, les conditions de la vie quotidienne (accès au logement et au travail par exemple) ou la participation au processus de décision politique. On voit bien ici apparaître le caractère multidimensionnel du progrès.

Enfin, une dernière dimension du progrès réside dans la soutenabilité, soit, pour faire court, dans l'évaluation du patrimoine, notamment environnemental. Cette évaluation pose des problèmes redoutables de mesure, dont on ne peut pas envisager la traduction en termes monétaires, au moins pour le moment. À noter également que le rapport propose, sur cette dernière dimension, des indicateurs d'alerte climatique et biologique, un point sur lequel il dépasse le strict domaine de la mesure des phénomènes pour entrer dans celui de la décision, ce dont on ne peut que se féliciter car la mesure n'est pas désincarnée, elle doit aboutir à des actions ${ }^{3}$.

L'ensemble de ces propositions suscite des réflexions et questions parfois contradictoires. À un premier niveau d'analyse, on peut se réjouir que des économistes se départissent de ce qui est souvent considéré comme leur forme de pensée unique et rejoignent ce que la sagesse populaire a exprimé depuis longtemps en affirmant que l'argent ne fait pas le bonheur.

Mais ce serait oublier que depuis très longtemps déjà, les spécialistes avaient reconnu les insuffisances du PIB. Les pères fondateurs, les créateurs des outils de la comptabilité nationale étaient bien conscients de ses limites. Ils ne voyaient en lui qu'un résumé comptable indispensable, sans lequel on serait bien démuni, mais certainement pas une mesure du progrès économique, même si, dans la situation de l'après-guerre, le développement quantitatif était une priorité évidente. Ils étaient également soucieux des problèmes d'inégalités et des questions de répartition ${ }^{4}$.

Ces outils ont été mal interprétés et le PIB, qui aurait pu ne rester qu'une variable des modèles économétriques, est devenu un indicateur privilégié de résultat, un objectif ; comme si les médecins faisaient $d u$ thermomètre l'indicateur unique de la bonne ou mauvaise santé. Et c'est à bon droit que l'on a critiqué depuis longtemps cet usage abusif. Au début, cette critique fut le fait des fractions les plus contestataires de l'opinion. C'était l'une des positions revendiquées en I968, mais, à l'époque, on ne voit pas d'autorité officielle qui l'aurait endossée.

Il aura donc fallu 40 ans - une génération - pour que ces idées, au départ minoritaires dans l'opinion, s'imposent peu à peu et pour que la position contestataire d'antan puisse devenir une vérité officielle, ce qui fournit une mesure du temps de latence de la diffusion des idées.

Une bonne partie des économistes avaient déjà échappé à ce piège et

3. Voir, à ce sujet, JOUVENEL Hugues (de). « La mesure du bien-être ». Futuribles, n 357, novembre 2009, pp. 3-4.

4. En ont témoigné, en leur temps, la création du Centre d'étude des revenus et des coûts, et celle du Centre de recherche, d'études et de documentation sur la consommation, qui visaient à compléter l'information donnée par le PIB et, plus généralement, la comptabilité nationale sur des sujets dont la Commission a souligné l'importance. 
étaient conscients de la nécessité de disposer d'autres instruments pour évaluer le progrès. Le rapport ne le fait pas apparaître dans la version « synthèse » qui, en excellente vulgarisatrice, évite les termes savants et les développements techniques. Mais il suffit de jeter un coup d'œil au rapport complet, aux documents de travail qui l'accompagnent et aux abondantes références qu'il comporte, pour se rendre compte que, derrière les idées simples exprimées par les recommandations, il y a une somme impressionnante de travaux scientifiques approfondis.

\section{Le progrès social vu par les seuls économistes}

Ces travaux sont largement ignorés de l'opinion publique qui ne voit dans les économistes que des monstres froids. Il existe, en France, une coupure, peut-être plus forte qu'ailleurs, entre les clercs et le peuple, un syndrome technocratique, et la Commission ne semble pas y avoir échappé. Les réflexions qu'elle a présentées résultent d'échanges entre experts, à huis clos, sans consultation de la société civile. Et pourtant, cette dernière était la première concernée puisqu'il s'agissait de rechercher les critères d'une juste répartition des biens, la nature des moyens à considérer pour assurer l'avenir de nos descendants, les conditions à remplir pour réaliser le progrès social.

Un autre cloisonnement, entre clercs celui-là, apparaît à la composition de la Commission. Elle ne comprend pratiquement que des écono- mistes. Pourtant, s'agissant de traiter du progrès social, on aurait pu s'attendre à y voir figurer de façon significative des psychologues ${ }^{5}$, des sociologues, des philosophes, voire des médecins ou des historiens. Certes, en ne faisant appel qu'à des spécialistes d'une même discipline, munis du même référentiel d'analyse, ils ont pu plus facilement identifier les sujets de discussion et arriver à un quasi-consensus. Mais n'ont-ils pas ainsi recréé une forme de pensée unique ? N'est-ce pas aussi une manifestation de l'ambition hégémonique de la théorie économique qui se sent capable d'appliquer ses outils d'analyse à bien d'autres situations que celles qui constituent son « cour de métier »?

On aurait pu envisager un fonctionnement de la Commission qui évite ce double cloisonnement, en élargissant sa composition et en accompagnant ses travaux d'un débat public sur les attentes des citoyens en matière de progrès économique et social. Évidemment, quand on lance ce genre de machine, on ne peut pas être sûr à l'avance de la trajectoire qu'elle prendra. En revanche, avec la méthode suivie, on pouvait dès le départ prévoir les grandes directions des résultats auxquels on aboutirait, et envisager sans risque la possibilité de tirer des conclusions opérationnelles assez rapidement et de lancer quelques idées susceptibles de faire choc dans l'opinion publique.

Mais ne serait-il pas opportun, cette première étape acquise, de poursuivre les réflexions faites, en élargissant le débat à partir des bases que

5. Certes, la Commission comprenait un psychologue, Daniel Kahneman. Mais ce dernier a effectué une bonne part de ses travaux sur les processus de décision, en liaison avec des économistes, et c'est en économie qu'il a obtenu son prix Nobel. 
constitue le rapport, et en le faisant sortir du champ clos des experts économistes ? Cela permettrait d'éviter qu'il ne se traduise finalement que par l'adjonction d'un chapitre supplémentaire dans les rapports des comptes nationaux. Ce genre d'issue sera déjà un progrès important, même s'il risque de se passer bien du temps avant que la répétition des informations fournies sur le sujet en entraîne la diffusion dans l'opinion et finalement la prise en compte par les décideurs.

\section{POUR ALLER PLUS LOIN}

Les publications de la Commission :

ALKIRE Sabina. The Copability Approach to the Quality of Life. Paris : 2008, 22 p. CMPEPS (Commission sur la mesure des performances économiques et du progrès social), INSEE (Institut national de la statistique et des études économiques), OCDE (Organisation de coopération et de développement économiques). Survey of Existing Approaches to Measuring Socio-Economic Progress. Paris : 2008, 58 p.

FLEURBAEY Marc. Beyond GDP: Is There Progress in the Measurement of Individual Well-Being and Social Welfare? Paris : 2008, 48 p.

FLEURBAEY Marc. Individual Well-Being and Social Welfare: Notes on the Theory. Paris : 2008, $36 \mathrm{p}$.

STIGLITZ Joseph, SEN Amartya et FITOUSSI Jean-Paul. Note problématique de la Commission sur la mesure des performances économiques et du progrès social. Paris : 2008, 43 p.

STIGLITZ Joseph, SEN Amartya et FITOUSSI Jean-Paul. Mesure des performances économiques et du progrès social réexaminée. Réflexions et vue d'ensemble des questions abordées. Paris : 2009, 72 p.

Le site Internet www.stiglitz-sen-fitoussi.fr/fr/documents.htm. 


\section{VIVRE EN 2040}

Comment vivrons-nous en 2040 ? Mano a Mano et Futuribles International présentent une série de quatre documentaires sur l'avenir de nos sociétés.

Bébé sur mesure, ventres à louer, voiture à la betterave ou ville verticale $100 \%$ bio, autant de scénarios possibles pour les trente prochaines années. Des chantiers sont en cours, des recherches annoncent des lendemains qui chantent ou qui déchantent.

" Science sans conscience "... On connaît la maxime. Aux promesses de la science, il faut ajouter les choix de société, les enjeux humains ou écologiques. Et quand il s'agit de se reproduire, de manger, de se déplacer ou d'habiter quelque part, tout est lié. Alors comment raconter le monde tel qu'il tournera en 2040 , loin de toute science-fiction et de tout fantasme ?

Ces quatre films interrogent chercheurs, scientifiques, médecins, cuisiniers, architectes ou encore urbanistes, et vont débusquer les signes avant-coureurs du monde tel qu'on l'envisage et tel qu'on voudrait le laisser à nos enfants.

Un voyage prospectif qui nous dit, au passage, que ce sont les décisions que nous prenons aujourd'hui qui dessinent les contours de ce que vivront les enfants de 2040 . Ces mêmes enfants qui, loin d'accepter en bloc I'héritage que nous leur laisserons, inventeront à leur tour d'autres futurs possibles.
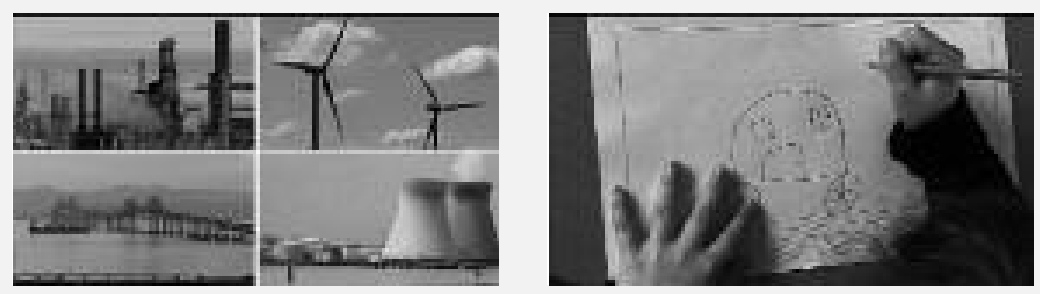

\section{LES QUATRE FILMS}

Naître en 2040 diffusé mardi 6 avril à 20 h35 Se déplacer en 2040 diffusé mardi 6 avril à 21h40 Manger en 2040 diffusé mardi 13 avril à 20 h35 Habiter en 2040 diffusé mardi 20 avril à 20 h35

\section{EN AVRIL SUR FRANCE 5}

